\title{
Response to Jin Hyun Kim Dynamics of Musical Expression
}

\author{
MINE DOĞANTAN-DACK \\ Middlesex University, London, UK
}

\begin{abstract}
This commentary focuses on three aspects of the experience of musical expressiveness as a dynamically emergent phenomenon: the nature of the experience itself, the nature of the dynamic forms of music, and the process of shaping music expressively in the act of music-making.
\end{abstract}

Submitted 2013 May 15; accepted 2013 May 30.

KEYWORDS: musical expression, forms of vitality, musical movement, kinaesthesis

IN the wake of the collapse of the Cartesian philosophy of mind in Western thought, much progress has been made in neuroscience over the last three decades in unravelling the nature and mechanisms of mental phenomena. Arguably the most powerful premise to emerge from this research is the inadequacy of dualistic conceptions that have severed the mind from the body, thinking from emotions, and the self from the rest of the world in mainstream philosophical and psychological thought for the greater part of the $20^{\text {th }}$ century. Recent research on the human brain carried out through advanced technological means provides overwhelming evidence to dissolve decisively these conceptual dualities, and to support the hypotheses that 'the mind' as the site of mental phenomena is fully embodied (Damasio, 2000; Edelman, 2007); that affective experiences including emotions constitute an integral background for cognitive processes (Damasio, 1994, 2000; LeDoux, 1999: Sousa, 1987); and that subjectivities are grounded in inter-subjective experiences (Bråten, 1998, 2009: Stern, 1985; Trevarthen, 1993). Concomitant with these developments has been a general shift of epistemological emphasis in contemporary psychology towards a concern with dynamic aspects of experience, such that conscious experience is regarded as grounded in the kinesthetic awareness of the dynamic processes unfolding in and with the body as it moves (Sheets-Johnstone, 2011; Stern, 1985). I therefore welcome Jin Hyun Kim's timely contribution which - in keeping with recent research in neuroscience and psychology - proposes a dynamic view of musical expression as an emergent phenomenon rooted in fundamental felt experiences associated with the movements of our own bodies and of other dynamic events. In her article, Kim identifies these basic felt experiences as 'forms of vitality', borrowing a notion from developmental psychologist Daniel Stern (2010).

In this commentary I should like to address three questions that are fundamental for Kim's proposed theoretical framework and also for research in musical expression. These are: 1) what does an experience of musical expressiveness consist of?; 2) what exactly are forms of vitality in relation to music and musical experiences?; and 3) what is involved in shaping music expressively? But first, a note on the originality of Kim's approach would be useful: while, to my knowledge, the conceptual coupling of music making (performer) and perception (listener) in the emergence of the experience of musical expressiveness has not been explored before, the individual components of the proposed theoretical framework are not entirely novel in empirical music psychological research. For instance, "how musical expressiveness emerges in the course of music-making", i.e. as a dynamic process, which is one of the central questions Kim poses, has been a core concern in research on expressive music performance. Indeed, some of this research deals with the origins of the dynamic processes, e.g. changing tempos and dynamic intensities, observed in the acoustical characteristics of musical performances, positing the dynamics and constraints of motor actions as just such an origin (e.g. Todd, 1992). Leech-Wilkinson's research on performance styles (2009) is also largely concerned with the dynamic nature and features of expressive music making. The idea that listening is not a passive reception of aural impressions but an active process involving bodily participation, which is another aspect of the framework proposed by Kim, has also been previously articulated in research (Cox, 2011; Cross, 2010; Godøy, 2003). Finally, while the introduction of the term "forms of vitality" into a discussion of musical expressiveness is a novel feature of her approach, as Kim conceptually aligns forms of vitality with the theory of dynamo-agogics proposed by Alexander Truslit, one can find precedents in expressive performance research also for this kind of conceptualization of musical expressiveness (e.g. Repp, 1996).

What does an experience of musical expressiveness consist of? I am very supportive of Kim's attempt to broaden the conceptual space of musical expressiveness beyond the expression of emotions, 
understood as discrete experiential categories with labels such as sadness, happiness, fear, anger, etc. Defined in this way, I do not think emotion is necessary for the creation and reception of expressiveness in music, and psychological research has arguably put too much emphasis on emotions in relation to other kinds of greatly varied experiential qualities that accompany the experience of musical expressiveness. What is necessary, however, for anyone - performer or listener - to experience music as expressive is a certain valorized felt affect, which does not necessarily lead to a named emotion. Kim's emphasis on the idea that music is regarded as expressive to the extent that the individual experiences it in terms of - or, through the lens of - an affective component is thus very apposite. For purposes of research, it is advisable not to treat musical expression as an ontological subcategory of emotional expression, as this pushes a wide range of other experiential phenomena related to musical expression that does not represent emotions out of the conceptual range of the researcher from the start. I am not convinced, however, that the experience of musical expression needs to be treated necessarily as an aesthetic experience, "a special form of musical experience not yet investigated in much detail within the scope of music and emotion research" in Kim's words. While some experiences of music's expressiveness can indeed have the characteristics of an aesthetic experience as defined by John Dewey and adopted by Kim, it is not an empirically established fact, nor is it conceptually necessary, that all experiences of musical expressiveness be aesthetic in nature: for example, humans can react to a short, fleeting moment of music they hear through a valorized affective response, where the "moment" thus experienced does not "reach an inclusive and fulfilling close" or "accumulate toward objective fulfillment" as in Dewey's theory of aesthetic experience. In reality, there exists a great variety of listening practices that do not correspond to the normative or idealized listening mode - "fixated listening" (Biddle, 2011) - promoted in musicology. What is needed is extensive research on how different listening modes influence the reception as well as the creation of expressiveness in musical practices. Perhaps the most serious issue regarding the theoretical framework Kim proposes, however, is the lack of cultural considerations: research indicates that the expressiveness of performed music is contingent on historical-cultural circumstances (LeechWilkinson, 2009), and that there are learned, idiomatic affective responses that humans display when engaging with music as part of their acquired cultural behaviour (e.g., Solís, 2004). We do not automatically respond to "foreign" musical idioms through such learned characteristic responses. Furthermore, descriptions people give of the effects of musical expressiveness are consistently mediated through culture-specific values (e.g., Feld, 1990), and I, for one, would be interested in seeing Kim's proposed theoretical framework develop in this direction, i.e. to incorporate the culture-specific evaluative responses that are an essential part of the ways people talk about their experiences of musical expressiveness.

What exactly are forms of vitality in relation to music and musical experiences? In her opening statement, Kim writes that her article "considers expressive forms of music as 'forms of vitality." Although the article does not make explicit where these forms inhere or whence they emerge, one gleans from the text that "expressive forms of music are conceived of as shaped forms of vitality", implying that they emerge during the act of music making, i.e. in performance. According to Stern (2010, p. 20), "concerning art, it is obvious that vitality dynamics is a fundamental aspect of performance in the time-based arts", yet the idea of forms of vitality as emergent in the act of music making confronts us with a kind of complexity that is not evident, for example, in dance performance. Given that forms of vitality are related to qualities associated with movement, the particular forms of vitality shaped by a dancer as she moves, thus creating "the dance", are one and the same as the movements of the dancer's body: in this regard, the dance and the dancer are one. In music, however, there are two different kinds of forms of vitality that are shaped concurrently, yet are not identical: those constituted by the movements of "the music", i.e. dynamic forms that emerge as we perceive tonal relationships unfold in time, and those constituted by our perception of the movements of the performer's body. We are far from understanding how exactly these two distinct dynamic sources of musical expressiveness might be related, giving rise to a unified experiential Gestalt. To give one example, in the Finale of Beethoven's Piano Trio Op. 1 No. 3 in C minor, an expressively aweinspiring moment unfolds between measures 170-172: in measure 170, the music cadences in F major with a crotchet-long root-position tonic chord in the piano, and an $\mathrm{F}$ in the violin. The piano part has a rest in the rest of measure 170 and in measure 171, while the violin sustains the $F$ from measure 170 to the middle of measure 172, when the piano enters with an arpeggiated root-position D-flat major chord: physically, the pianist is still, or poised through measure 171, i.e. does not display any notable external movement between mm. 170 and 172, and the violinist is only moving to sustain one note - in terms of the forms of vitality that are being shaped through the bodies of the performers, there is minimal physical activity, and hence minimal movement, force or directionality displayed in physical space. With the onset of measure 172, however, we realize that between measures 170 and 172, a magical musical movement has taken place in pitch space, with a definite sense of force, temporal unfolding and directionality, to a location that is not exactly very near: a movement embodied as a particular form of vitality within a particular kind of tonal pitch space. The experience of the expressiveness of this 
passage emerges from both kinds of forms of vitality unfolding simultaneously. Furthermore, it is not difficult to see that due to the different gestural affordances of each kind of musical instrument, the emergent forms of vitality giving rise to experiences of expressiveness would be different when a given melody is played on a piano and on a flute, for example, even as the dynamic qualities of the melodic movement might remain identical (I say "might" here since the same notes played on the same kind of instrument can acquire different forms of vitality when played in different tempos, etc.). It is precisely the dynamic relationship between our experience of the movements in physical space of humans making music and the movements of music in tonal space that needs to be investigated and incorporated into Kim's theoretical framework if the concept of "forms of vitality" is to be applied effectively in the context of musical expressiveness, and the mechanisms responsible for its emergence are to be investigated. In other words, we need extensive research to understand what the forms of vitality emerging from his/her bodily movements in making music feel like for the performer; how these relate to the experience of emergent 'musical' forms of vitality generated by the experience of tonal relationships; and how both of these relate to the listener's felt experience of forms of vitality as a unified Gestalt in an experience of musical expressiveness. Furthermore, since in different musical cultures, pitch spaces are configured differently, creating different motional affordances, the culturalspecificity of musical forms of vitality should be part of any research agenda in musical expressiveness. In this connection, the distinction Sheets-Johnstone (2011, p. 305) makes between "form values" and "animate values" could be a useful tool, since "unlike form values, in the strict sense of morphology, [animate values] are (or can be) differently modified by culture."

What is involved in shaping music expressively in the act of music-making? According to Kim, the act of music-making, as well as the act of listening, both involve a process of "kinaesthetic simulation". It is not difficult to understand how the listener's experience of expressiveness may involve kinaesthetic images accompanying motor simulation: there is evidence in research, some of which is also cited by Kim, suggesting that such a process indeed takes place in engaging with music as listeners (Godøy, 2010; Leman, 2010). However, it is not entirely clear what it is exactly that the performer simulates: is it the experienced dynamic relationships between the tones, i.e. musical movement? If so, is there a one-to-one correspondence between musical movement and kinesthetic movement? Such important questions are not addressed in Kim's article, leaving the proposed theoretical framework conceptually in need of refinement. Furthermore, researchers need to be wary of drawing facile analogies between the activity of making music and that of listening: while listening may indeed involve a certain co-shaping process, we are far from confidently asserting in research terms that there is a substantial similarity in terms of the bodily and affectively felt qualities of the forms of vitality as experienced by the person actively producing the musical sounds and the person observing/listening to this process of shaping. It is not a clearly established fact that the intense physicality of making music constitutes a difference only in degree in comparison to the physicality of listening to music: performers, unlike listeners, come to know and represent the expressive details of the music they play kinaesthetically. Information regarding expressiveness is imprinted in their musculature, and committed to long-term memory, unlike in the case of non-performing listeners. Hence, extensive research is needed to further articulate the exact nature of the co-shaping process Kim puts forward as part of the emergent experience of musical expressiveness.

Jin Hyun Kim presents a theoretical approach to musical expressiveness that promises to go beyond the mainstream conceptualization of the phenomenon as a subspecies of emotional expression. Her orientation towards the inter-subjectively shared and understood forms of vitality as a basis for exploring expressiveness in music is a novel approach. Empirical investigations of her hypotheses will no doubt reveal much about the mysteries and magic of the universal human capacity for expressive music-making.

\section{REFERENCES}

Biddle, I. (2011). Listening, consciousness, and the charm of the universal: What it feels like for a Lacanian. In: D. Clarke \& E. Clarke (Eds.), Music and Consciousness: Philosophical, Psychological, and Cultural Perspectives. New York: Oxford University Press, pp. 65-78.

Bråten, S. (Ed.) (1998). Intersubjective Communication and Emotion in Early Ontogeny. Cambridge: Cambridge University Press.

Bråten, S. (2009). The Intersubjective Mirror in Infant Learning and Evolution of Speech. Philadelphia PA: John Benjamins Publishing.

Cox, A. (2011). Embodying music: Principles of mimetic hypothesis. Music Theory Online, Vol. 17, No. 2. URL: www.mtosmt.org/issues/mto.11.17.2/mto.11.17.2.cox.html 
Cross, I. (2010). Listening as covert performance. Journal of the Royal Music Association, Vol. 135, Supplement 1, pp. 67-77.

Damasio, A. (2000). The Feeling of What Happens: Body, Emotion and the Making of Consciousness. London: Vintage.

Damasio, A. (1994). Descartes’ Error. New York: Harper Collins.

Edelman, G. (2007). Second Nature: Brain Science and Human Knowledge. London: Yale University Press.

Feld, S. (1990). Sound and Sentiment: Birds, Weeping, Poetics, and Song in Kaluli Expression. Philadelphia: University of Pennsylvania Press.

Godøy, R.I. (2010). Gestural affordances of musical sound. In: R.I. Godøy \& M. Leman (Eds.), Musical Gestures: Sound, Movement and Meaning. New York: Routledge, pp. 103-125.

Godøy, R.I. (2003). Motor-mimetic music cognition. Leonardo, Vol. 36, No. 4, pp. 317-319.

LeDoux, J. (1999). The Emotional Brain: The Mysterious Underpinnings of Emotional Life. London: Phoenix.

Leech-Wilkinson, D. (2009). The Changing Sound of Music: Approaches to Studying Recorded Musical Performance. London: CHARM.

URL: http://www.charm.kcl.ac.uk/studies/chapters/intro.html

Leman, M. (2010). Music, gesture and the formation of embodied meaning. In: R.I. Godøy \& M. Leman (Eds.), Musical Gestures: Sound, Movement and Meaning. New York: Routledge, pp. 126-153.

Repp, B. (1996). Dynamics of expressive piano performance: Schumann's "Träumerei” revisited. Journal of the Acoustical Society of America, Vol. 100, No. 1, pp. 641-650.

Sheets-Johnstone, M. (2011). The Primacy of Movement. Philadelphia: John Benjamins Publishing.

Solís, T. (Ed.) (2004). Performing Ethnomusicology: Teaching and Representation in World Music Ensembles. Berkeley: University of California Press.

Sousa, R.D. (1987). The Rationality of Emotion. Cambridge, MA: MIT Press.

Stern, D. (1985). The Interpersonal World of the Infant: A View from Psychoanalysis and Developmental Psychology. New York: Basic Books.

Stern, D. (2010). Forms of Vitality: Exploring Dynamic Experience in Psychology and the Arts. Oxford: Oxford University Press.

Todd, N. (1992). The dynamics of dynamics: A model of musical expression. Journal of the Acoustical Society of America, Vol. 91, No. 6, pp. 3540-3550.

Trevarthen, C. (1993). The self born in intersubjectivity: An infant communicating. In: U. Neisser (Ed.), The Perceived Self: Ecological and Interpersonal Sources of Self-Knowledge. New York: Cambridge University Press, pp. 121-173. 